\title{
Stroke Segmentation in Infrared Reflectograms
}

\author{
Paul Kammerer, Georg Langs, Robert Sablatnig, and Ernestine Zolda \\ PRIP - Vienna University of Technology, \\ Favoritenstrasse 9/183/2, A-1040 Vienna, AUSTRIA \\ \{paul, langs, sab, zolda\}@prip.tuwien.ac.at
}

\begin{abstract}
An algorithm for the automatic segmentation of strokes in underdrawings - the basic concept of the artist - in ancient panel paintings is presented. The purpose of the stroke analysis is the determination of the drawing tool used to draft the painting. This information allows significant support for a systematic stylistic approach in the analysis of paintings. Up to now, this analysis has been made by naked eye examination only, and the restricted human optical retentiveness complicated the comparison of different underdrawings with respect to drawing tools and stroke characteristics. Stroke segmentation in painting is related to the extraction and recognition of handwritings, therefore similar techniques to segment the strokes from the background incorporating boundary information are used. Following the segmentation, the approximation of the stroke boundary by a closed polygon done based on active contours. Results of the algorithms developed are presented for both test panels and real reflectograms.
\end{abstract}

\section{Introduction}

Interdisciplinary projects between computer based imaging technologies and the field of art history brought new aspects in both of the fields. While art historians benefit from new objective analysis methods and improved efficiency due to computer based solutions, for technicians a new field of application was opened, which requires the adaptation and development of algorithms to the specific needs in art history.

Image processing methods are used for the corrections of distortions caused by the acquisition system, for stitching together detail images into a complete image (mosaicing) [1], for improving the visual image quality by contrast enhancement methods [2] or to restore the color of paintings [3]. These visualization methods are already state of the art in museum imaging technology [4].

Existing publications in the field of image analysis of paintings deal with automatic pattern recognition methods to assign a restricted drawings to the work of a certain artist [5] or the examination of the personal style of a painter based on the "structural significance" of brush strokes [6]. Tanaka et al. in [7] present tools for the extraction of compositional information from paintings. A brief overview about image recognition techniques that can be used for the 


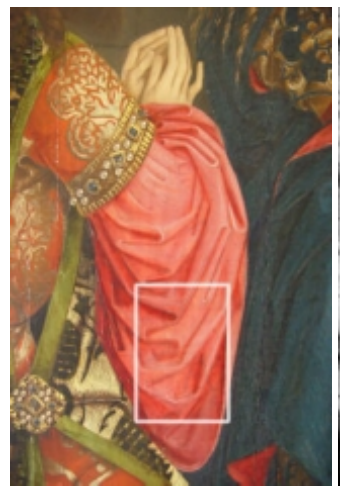

(a)

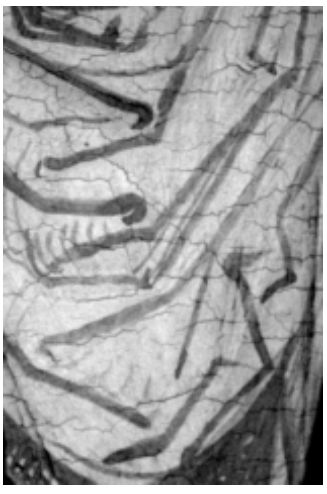

(b)

Fig. 1. "Adoration of the Kings", master of the Schottenstift (1470): (a) color image (b) image taken in the near IR range (700nm-900 nm)

classification of paintings is given in [8]. Mansfield et al. [9] used classification methodologies to analyze multi-spectral near-IR images of 16th century drawings.

The aim of our work is to develop methods for analysis of digital images of underdrawings in Medieval and Renaissance paintings to identify drawing tools like brush or chalk in order to investigate e.g. the authorship or to identify copies. Underdrawings are the basic concept of an artist when he starts the creation of a panel painting. To visualize underdrawings, which are not penetrable in the visual light spectrum since they are covered by the painting layers, the images are taken by a state of the art technology infrared camera system. This method is known as infrared reflectography, and the images produced as infrared reflectograms [10]. An infrared reflectogram showing the underdrawing of a part of a painting from the "Master of the Schottenstift" (a famous Austrian painter in the 15th century) is given in Figure 1(b).

The analysis of underdrawings is performed primarily by visual inspection of the reflectograms only. But the restricted human optical retentiveness complicates the comparison of different underdrawings concerning drawing tools, drawing materials, and stroke characteristics. In a first step towards the identification of artist specific characteristics in handling drawing tools, methods to segment strokes and lines of brush and chalk are developed. These methods are based on the observation that prominent characteristics of drawn strokes are variations of shape and of the intensity in the drawing direction.

In this paper we present a two step approach to stroke segmentation. In the first step an edge based segmentation method according to Doermann [11] is applied, to get a polygonal approximation of the boundary of stroke segments (Section 2.1). In the second step the polygon is used as an initialization for an active contour method, which adapts the polygon more precisely to the stroke boundary and yields an accurate identification of the boundary (Section 2.2). The method will be demonstrated on test panels with strokes of brush and 
chalk applied by a restorer and a sample reflectogram of an original painting (Section 3).

\section{Edge Based Stroke Segmentation in Works of Art}

Stroke segmentation in painting is related to the extraction and recognition of handwriting [12]. Letters and words in Western languages and symbols or signs in Chinese or Japanese languages are built of manually drawn strokes or lines. Many approaches start with thresholding and thinning methods. While these methods are fast and save resources, valuable information for a more detailed analysis of strokes requires an approach that also incorporates boundary information [11]. We used Doermann's segmentation algorithm in Step I, since it provides both, the boundary of a stroke and intensity profiles, which will be used to characterize strokes.

\subsection{Step I - Segmentation}

The segmentation procedure consists of the following three steps which are applied sequentially. Figure 2 sketches the steps of the method.

1. Edge detection: The edge detection step (see Figure 2(a)) provides a set of candidate edge pixels (edgels) $E_{i}(x, y)$, which are part of the strokes contour. The gradient vector $g_{i}=\left[\delta g_{i} / \delta x, \delta g_{i} / \delta y\right]^{T}$ assigned to an edgel $E_{i}$ is computed using the Canny edge detector with parameters: $\sigma$ (Gaussian filter as to choose the scale), $t h_{l o}$ and $t h_{h i}$ (low and high hysteresis threshold).

2. Building cross sections: Based on the hypothesis that (1) edgels lie on the boundary of a stroke and (2) the gradient vectors show in opposite directions (i.e. the angular difference between the gradient vector is above a threshold $\left.t h_{\text {opp }}\right)$, the procedure groups the set of edgels into corresponding pairs. For each edgel $E_{i}$ a corresponding edgel from a set of neighborhood edgels $E_{j}$ (within distance $t h_{\text {edist }}>=\left|\begin{array}{ll}E_{i} & E_{j}\end{array}\right|$ ) is chosen which minimizes the absolute difference $t h_{\text {ang }}=a b s\left(\theta_{1} \quad \theta_{2}\right) . \theta_{1}$ and $\theta_{2}$ is the angle difference between the normal to the gradient at position of $E_{i}$ and $E_{j}$ respectively and the cross section $C_{I}$, i.e. the connection of $E_{i}$ and $E_{j}$. Figure 2(b) shows two cross section candidates $C_{1}$ and $C_{2}, C_{1}$ is the one with smaller $D$.

3. Grouping of cross sections: The final step of the segmentation algorithm builds groups of cross sections that represent a stroke segment. Starting from one cross section $C_{1}$, neighbored cross sections $\left(C_{2}\right)$ are traced in both directions normal to the starting cross section, if they fulfill the following properties: (1) the orientation difference of two neighbored cross sections lies within a threshold $t h_{\text {ang } 1}(2)$ the length difference lies within a threshold $t h_{l e n}$ and (3) the center of the neighbored cross section is located within a search window of the center of the starting cross section, defined by $t h_{a n g 2}$ and $t h_{\text {dist }}$. Figure $2(\mathrm{c})$ illustrates the search window). The end of a stroke segment is defined by the cross section, which lacks a neighbored cross section that meets the grouping criteria. Closed 


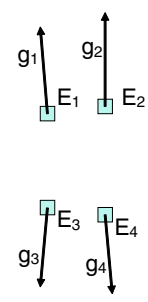

(a)

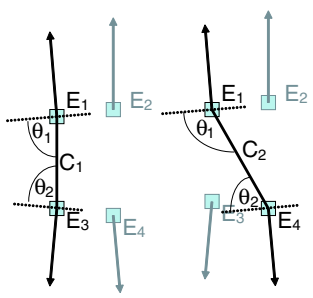

(b)

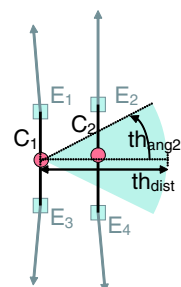

(c)

Fig. 2. Segmentation: (a) detected edgels and gradients (b) two options of building cross sections (c) search window of the grouping procedure

polygons connecting the endpoints of the cross sections define the boundary of the stroke segments.

\section{$2.2 \quad$ Step II - Boundary Refinement}

The approximation of the stroke boundary by a closed polygon is refined by "snakes", a method based on active contours [13].

After determining the first principal component of the edgel distribution i.e. approximating the direction of the stroke, the contour is split into two sides that are treated separately. Let $E=\left(E_{1}, E_{2}, \ldots, E_{n}\right)$ be the vector of edgels defining one side of the stroke. A set of gray value profiles $\mathbf{P}=\left[p_{1}, p_{2}, \ldots, p_{m}\right]$ where $m \approx\left\|E_{n} \quad E_{1}\right\|$, each lying orthogonal to the direction of the stroke, is extracted from the image. The profiles are orthogonal to the axis of the stroke and lie at equidistant positions. Figure 3(a) illustrates the edgels and the profiles perpendicular to the stroke axis. The matrix built by the profiles $\mathbf{P}(x, y)$ with $(x, y) \in[0, n] \times[0, m]$ is viewed as a distribution of gray values. The snake moves through this domain to minimize an energy functional determined by inner parameters controlling rigidity and tension of the snake and an external energy influenced by $\mathbf{P}(x, y)$. It is generated by generalized gradient vector flow in order to provide accurate and fast convergence to boundary concavities.

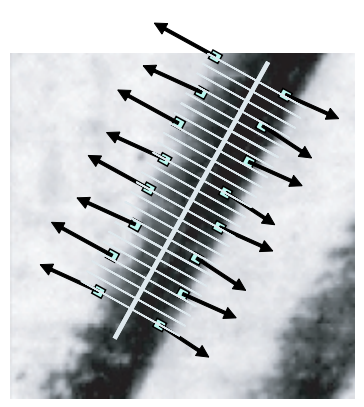

(a)

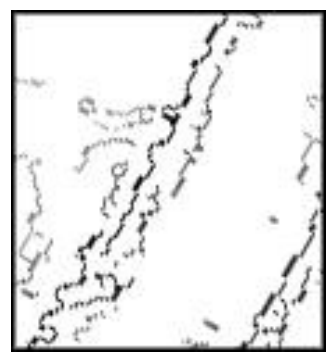

(b)

Fig. 3. Refinement: (a) edgels and gray value profiles (b) edge map 
The external energy is determined by a weighted edge map

$$
f(x, y)=\operatorname{sign}(c(x, y)) * \cdot(c(x, y)+s(x, y)+m(x, y))
$$

where $*$ denotes pixelwise multiplication and in which the outer continuous boundary of the stroke is emphasized. This is due to defining $c$ as the accumulation of responses of Canny edge detectors with different hysteresis thresholds which provide robustness and $s$ as the output of a Sobel operator that enhances edges with gray level gradient pointing away from the axis. $m$ is a function that determines whether a point lies on a connected edge that crosses the polygon defined by $E$. The edgemap calculated calculated from Figure 3(a) can be seen in Figure 3(b). By initializing the snake on the approximation given by $E$ the method yields an accurate identification of the stroke boundary and valuable information about the boundary roughness. Contour estimates with different levels of elasticity provide descriptive information by means of deviation against each other. For more details please refer to [14].

\section{Experiments on Test Panels and Reflectogram}

Image Acquisition: The test panels are digitized using a flat-bed scanner with an optical resolution of $1200 \mathrm{dpi}$. The images of the underdrawing are digitized by a Sony Night-Shot camera in the near infrared range within a spectral range between $700 \mathrm{~nm}$ and $1000 \mathrm{~nm}$ into a 1712 by 2560 image matrix at a resolution of about 500dpi.

Preprocessing/Eliminating Craquelés: Structural noise which occur in original paintings is caused by the aging process and results in craquelé. Figure 4(a) shows a detail of an underdrawing with craquelé occurring as thin horizontal branching lines. We used a morphological method according to [15], to remove the craquelé while preserving the strokes as accurately as possible. Details of the method are presented in [16]. The restored image is depicted in Figure 4(b).

Test Panels: Experiments have been performed on test panels showing strokes applied with different drawing tools. The panels are prepared by an expert without disturbing craquelé and scanned under controlled acquisition conditions. The panels are primarily used for testing the segmentation algorithm and estimating the parameters. The algorithm depends on the one hand on the edge detection result, i.e. the parameters of the Canny detector are choosen to obtain a continuous boundary of the strokes, and on the other hand on a predefined stroke width, with corresponds to the search window to build pairs of edgels $\left(t_{\text {edist }}\right)$.

Figure 5 shows a series of chalk strokes (a) applied in top down direction and brush strokes (b) applied in bottom up direction. While both brush strokes boundaries (e) are smooth, the left and right boundary of a chalk strokes (c) varies significantly. The segmentation algorithm is applied to images, resized by a factor of 0.25 using bilinear interpolation, in order to speed up the building of the cross sections and the grouping process. This reduction also smoothes the boundaries as well as the variation of the gray-values especially on chalk strokes. 


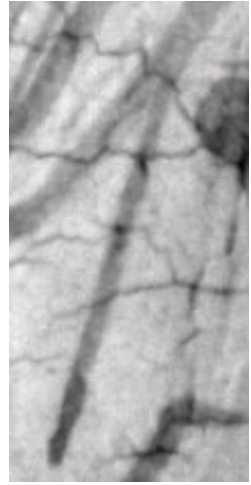

(a)

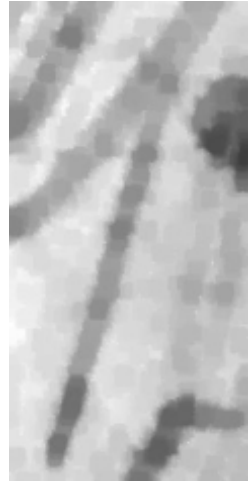

(b)

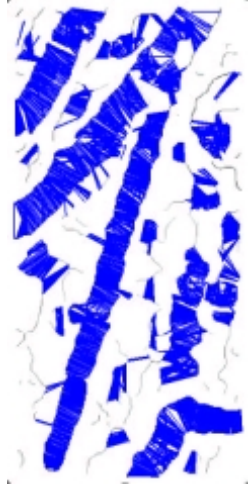

(c)

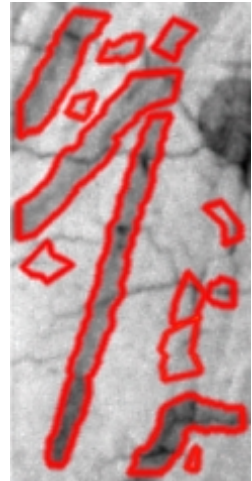

(d)

Fig. 4. Detail from an IR reflectogram showing strokes of an underdrawing and craquelés (a), the image after morphological reconstruction (b), cross sections (c) and the final segmentation of the strokes $(d)$.

Figure 5(d) and (f) show the edges resulting from edge detection, and the cross sections produced ( $d=15$ pixel). Figure $5(\mathrm{~g})$ and $(\mathrm{h})$ eventually show the snakes adapted to the boundary. By using two different, a low ("+"-line) and a high ("-"-line) energy value, the differences of the two resulting snakes describe the roughness of the boundary. You can observe highly overlapping snakes in both borders of the brush stroke and differences in the chalk stroke.

Reflectograms of Original Paintings: The second type of test has been performed on infrared reflectograms taken from original panel paintings (see Figure 4. The craquelés have been successfully removed by the morphological filtering that only the strokes remain (Figure 4(b)). The segmentation was applied to the images resulting from the morphological filtering. Figure 4 (d) shows the grouped cross-sections and (e) the polygonal boundary which corresponds with the boundary of the strokes in the original image. Some of the edgels which are not part of a stroke boundary, but fulfill the grouping criteria, are grouped into stroke segments (see Figure 4(d)). An additional processing step will eliminate these artifacts e.g. by applying a minimal segment length criteria.

\section{Conclusion}

The segmentation algorithm presented in this paper successfully detects the boundary of strokes represented as a closed polygon in test panels as well as in underdrawings of original paintings. The refinement of the boundary provides sufficient accuracy to distinguish between different boundary of drawing tools like brush and chalk.

We have shown how methods used in handwriting analysis can be adapted for use in painting analysis. The stroke segmentation method followed by boundary refinement is a first step towards the identification of drawing tools in under- 


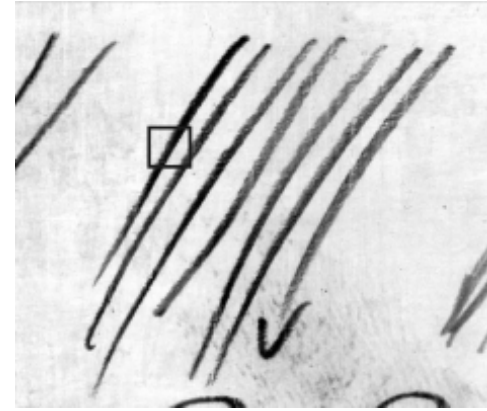

(a)

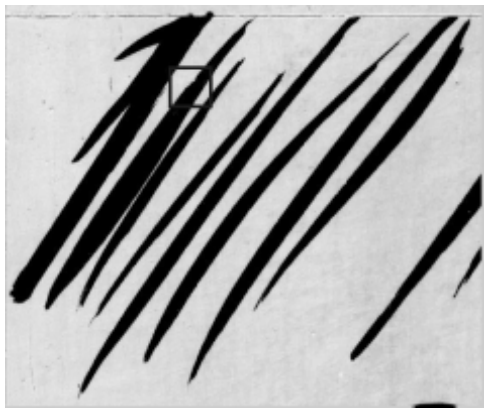

(b)

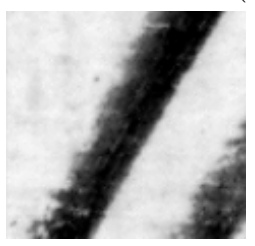

(c)

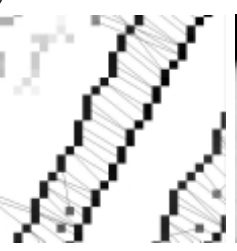

(d)

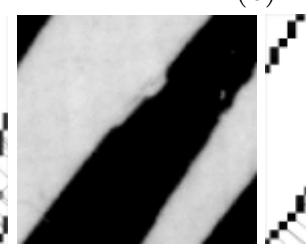

(e)

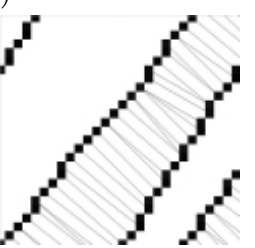

(f)

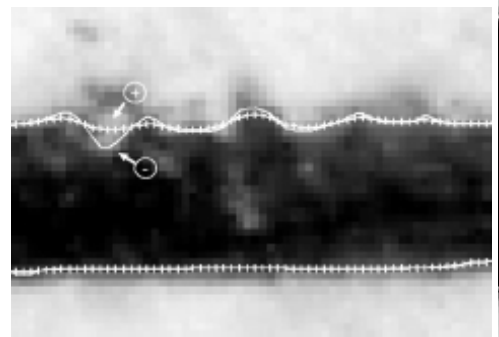

(g)

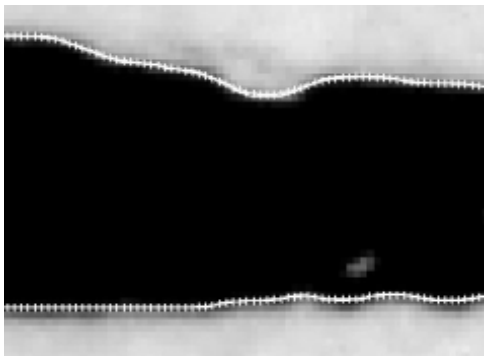

(h)

Fig. 5. Test panel with (a) chalk strokes and (b) brush strokes. (c),(e) details from (a),(b) showing differences in strokes boundary (d),(f) cross sections as part of the segmentation process; (g),(h) snake refinement

drawings. Based on the segmentation, features to characterize the boundary of strokes can be obtained in order to investigate e.g. the authorship of paintings according to the artist himself or his workshop.

Experiments with underdrawings of original paintings to test the algorithms against noise and outliers, with the goal to improve the robustness, will be part of future research. Further the approach will be extended to segment overlapping and crossing stroke formations as e.g. reported in [17], to investigate the intensity variations and to analyze the characteristic of stroke endings.

Acknowledgment: We would like to thank Allan Hanbury and Norbert Brändle for assistance in the field of mathematical morphology. This work was supported by the Austrian Science Foundation (FWF) under grants P15471-MAT and P14445-MAT. 


\section{References}

1. J.-M.Chassery W.Puech, A.G.Bors and I.Pitas. Mosaicing of paintings on curved surfaces. In Third IEEE Workshop on Applications of Computer Vision (WACV'96), pages 44-49, 1996.

2. D. Lagunovsky, M. Frucci, and G.S. di Baja. Image processing tools for fresco restoration. In 14th ICPR, volume 3, pages 326-329, 2000.

3. M. Pappas and I. Pitas. Digital color restoration of old paintings. Trans. On Image Processing, 9(2):291-294, 2000.

4. J.R.J. van Asperen de Boer. Infrared reflectography and computer image processing. New alternatives. In Le Dessin Sousjacent dans la Peinture, Coll. IX, pages 267-273, 1993.

5. S. Kroner and A. Lattner. Authentication of free hand drawings by pattern recognition methods. In 14th ICPR, volume 1, pages 462-464, 1998.

6. Robert Sablatnig, Paul Kammerer, and Ernestine Zolda. Hierarchical classification of painted portraits using face- and brush stroke models. In 14 th ICPR, Brisbane, Australia, August 17-20, 1998.

7. S. Tanaka, J. Kurumizawa, S. Inokuchi, and Y. Iwadate. Composition analyszer: support tool for composition analysis on paintin masterpieces. Knowledge-Based Systems, 13:459-470, 2000.

8. H. J. van den Herik and E. O. Postma. Future Directions for Intelligent Systems and Information Sciences, chapter Part II, Discovering the visual signature of painters. Springer, 2000.

9. J.R. Mansfield, M.G. Sowa, C. Majzels, C.Collins, E. Cloutis, and H.H. Mantsch. Near infrared spectroscopic reflectance imaging: supervised vs. unsupervised analysis using an art conservation application. Vibrational Spectroscopy, 19:33-45, 1999.

10. J.R.J. Van Asperen de Boer. Infrared Reflectography. - A Contribution to the Examination of Earlier European Paintings. PhD thesis, Univ. Amsterdam, 1970.

11. D.S. Doermann and A.Rosenfeld. Recovery of temporal information from static images of handwriting. International Journal of Computer Vision, 52(1-2):143164, 1994.

12. R. Plamondon and S.N. Srihari. On-line and off-line handwriting recognition: A comprehensive survey. Trans. on Pattern Analysis and Machine Intelligence, 22(1):63-84, 2000.

13. Chenyang $\mathrm{Xu}$ and Jerry L. Prince. Snakes, shapes and gradient vector flow. IEEE Transactions on image Processing, 7(3):359-369, March 1998.

14. G. Langs, H. Bischof, and P.L. Peloschek. Automatic quantification of destructive changes caused by rheumatoid arthritis. Technical Report 79, Vienna University of Technology, Pattern Recognition and Image Processing Group, 2003.

15. J. Serra. Les treillis visqueux. Technical Report N-51/99/MM, CMM, Ecole des Mines de Paris, 1999.

16. A. Hanbury, P. Kammerer, and E. Zolda. Painting crack elimination using viscous morphological reconstruction. appears in 12th Intl. Conf. on Image Analysis and Processing, ICIAP2003.

17. E. L'Homer. Extraction of strokes in handwritten characters. Pattern Recognition, 33(7):1147-1160, 1999. 